\title{
Simple multimodal optical technique for evaluation of free/bound water and dispersion of human liver tissue
}

Isa Carneiro

Sónia Carvalho

Rui Henrique

Luís Oliveira

Valery V. Tuchin 


\title{
Simple multimodal optical technique for evaluation of free/bound water and dispersion of human liver tissue
}

\author{
Isa Carneiro, ${ }^{a}$ Sónia Carvalho, ${ }^{a}$ Rui Henrique, ${ }^{\mathrm{a}, \mathrm{b}}$ Luís Oliveira, ${ }^{\mathrm{c}, \mathrm{d}, \star}$ and Valery V. Tuchin ${ }^{\mathrm{e}, \mathrm{f,g}}$ \\ aportuguese Oncology Institute of Porto, Department of Pathology and Cancer Biology and Epigenetics Group-Research Centre, Porto, Portugal \\ ${ }^{b}$ Institute of Biomedical Sciences Abel Salazar-University of Porto, Department of Pathology and Molecular Immunology, Porto, Portugal \\ 'Polytechnic of Porto, School of Engineering, Department of Physics, Porto, Portugal \\ ${ }^{\mathrm{d} C}$ Centre of Innovation in Engineering and Industrial Technology, ISEP, Porto, Portugal \\ eSaratov State University (National Research University of Russia), Research-Educational Institute of Optics and Biophotonics, Saratov, Russia \\ ${ }^{f}$ Precision Mechanics and Control Institute of the Russian Academy of Sciences, Laboratory of Laser Diagnostics of Technical and Living Systems, \\ Saratov, Russia \\ g/TMO University, Laboratory of Femtomedicine, St. Petersburg, Russia
}

\begin{abstract}
The optical dispersion and water content of human liver were experimentally studied to estimate the optical dispersions of tissue scatterers and dry matter. Using temporal measurements of collimated transmittance $\left[T_{\mathrm{c}}(t)\right]$ of liver samples under treatment at different glycerol concentrations, free water and diffusion coefficient $\left(D_{\mathrm{gl}}\right)$ of glycerol in liver were found as $60.0 \%$ and $8.2 \times 10^{-7} \mathrm{~cm}^{2} / \mathrm{s}$, respectively. Bound water was calculated as the difference between the reported total water of $74.5 \%$ and found free water. The optical dispersion of liver was calculated from the measurements of refractive index (RI) of tissue samples made for different wavelengths between 400 and $1000 \mathrm{~nm}$. Using liver and water optical dispersions at $20^{\circ} \mathrm{C}$ and the free and total water, the dispersions for liver scatterers and dry matter were calculated. The estimated dispersions present a decreasing behavior with wavelength. The dry matter dispersion shows higher RI values than liver scatterers, as expected. Considering $600 \mathrm{~nm}$, dry matter has an RI of 1.508, whereas scatterers have an $\mathrm{RI}$ of 1.444 . These dispersions are useful to characterize the RI matching mechanism in optical clearing treatments, provided that $\left[T_{c}(t)\right]$ and thickness measurements are performed during treatment. The knowledge of $D_{\mathrm{gl}}$ is also important for living tissue cryoprotection applications. $\odot 2017$ Society of Photo-Optical Instrumentation Engineers (SPIE) [DOI: 10.1117/1.JBO.22.12.125002]
\end{abstract}

Keywords: human liver tissue; refractive index; free and bound water; optical clearing; diffusion coefficient; cryoprotection.

Paper 170637RR received Sep. 30, 2017; accepted for publication Nov. 15, 2017; published online Dec. 5, 2017.

\section{Introduction}

The optical properties of biological tissues depend on their internal composition and each particular tissue has a specific combination of the various biological components. Soft tissues contain mainly cells and their organelles, as well as proteins and fibers of the extracellular matrix, which are distributed throughout a liquid ground substance, commonly designated by cell cytoplasm and interstitial fluid (ISF), respectively. ${ }^{1-5}$ The ISF contains mainly water and a small amount of dissolved salts, proteins, and organic compounds. ${ }^{5-7}$ The water content in these fluids will condition light scattering, as well as absorption in UV and IR, due to the refractive index (RI) mismatch between the ISF and other tissue components. ${ }^{5}$

Water in biological tissues can be classified as bound or free. Bound water is tightly connected to the other tissue components, commonly designated as dry matter, and requires a strong or long-term stimulation to convert into free water. Free water, on the other hand, can be found in the ISF or inside the cells. It can move from one place to another inside the tissue, or to the outside, if appropriate stimulation is provided. ${ }^{8,9}$ The combination of dry matter and bound water in tissues is commonly designated as scatterers.

Scatterers in biological tissues have higher RI values when compared to the RI of the ISF. At $589.6 \mathrm{~nm}$, the RI values for the ISF range approximately between 1.35 and 1.37 , and the ones for scatterers usually range between 1.39 and $1.47,{ }^{5}$ or even higher as 1.58 for dry muscle proteins ${ }^{6}$ or 1.6 for skin melanin. ${ }^{5}$ No perceptible physical boundaries between tissue components can be understood in a macroscopic scale, meaning that biological tissues can be considered as a continuous material with spatial variations in RI. ${ }^{5}$ Such high RI mismatch between components creates the strong light scattering properties that most soft tissues present. ${ }^{5}$

To reduce light scattering in tissues, the optical clearing (OC) technique has been investigated in the past years. ${ }^{5,10-14}$ During short-time OC treatments, only the free water in tissues will flow out (dehydration mechanism ${ }^{15}$ ) to allow the optical clearing agent (OCA) to flow in and create the RI matching mechanism. ${ }^{15}$ The determination of the diffusion coefficients of OCAs and water that are characteristic to these mechanisms is highly important. Some OCAs that have been studied are glucose,${ }^{12,16}$ glycerol, ${ }^{11,17}$ dimethyl sulfoxide, mannitol, propylene glycol, and x-ray contrast, such as Trazograph ${ }^{\mathrm{TM}}$ and Omnipaque $^{\text {TM }} \cdot{ }^{14,18}$ Such studies have demonstrated a significant decrease in tissue scattering properties and an increase in tissue depth and image contrast when using imaging techniques. ${ }^{10}$

A variation in the RI of tissue's ISF will occur due to the exchange between the free water in the tissue and the OCA in the treating solution. The RI of scatterers, on the other 
hand, will remain unchanged during OC treatments. If the RI of scatterers is known, or can be calculated, it is possible to estimate the RI change in the ISF and characterize the RI matching mechanism in the tissue. ${ }^{19}$ Gladstone and Dale equation is commonly used to calculate the wavelength dependence of tissue RI from the RI of tissue components. ${ }^{20}$ On the other hand, if the total and free water content in the tissue are known, such equation can also be used to calculate the RI of the dry matter or scatterers in the tissue. The knowledge of the total and free water content in biological tissue is consequently of high importance to quantify light scattering and absorption and to plan efficient OC treatments to reduce light attenuation. The refractive dispersions of tissue scatterers and dry matter are also useful for treatment planning or even for diagnostic purposes.

Since free and bound water contents have been found to be different between normal and malignant tissues, ${ }^{11,21}$ the knowledge of these values is also of great importance to consider them as a marker of cancer progression in general. Quantification of free/bound water ration in a particular tissue is urgently needed. Liver tissue is not yet fully studied from this point of view. In the literature, we can find only some very useful data on liver optical properties dependence on induced changes in RI and osmolarity for action of some solutes. ${ }^{22}$ Therefore, it was our objective to determine free water content and calculate the RI dispersion of human liver tissue, of its dry matter and scatterers by using as a probe molecular glycerol in water solutions with a controllable osmolarity. Since we used glycerol/water solutions, we wanted also to study the diffusion properties of this molecule in human liver. To perform this research, we have made various experimental studies, including direct measurements of tissue RI for a few wavelengths as described in Sec. 2.

\section{Materials and Methods}

Different methods were used to perform this research with human liver. Section 2.1 presents a description of the tissue samples and their preparation. Sections 2.2 and 2.3 describe the experimental methodology used to measure the RI of liver and the spectral collimated transmittance $\left(T_{\mathrm{c}}\right)$ during OC treatments. Section 2.4 describes the calculation procedure to estimate the free water in liver, and the RI dispersion for the tissue, its dry matter, and scatterers.

\subsection{Tissue Samples}

Human adult liver contains $74.5 \%$ of water, while the remaining $25.5 \%$ represents a combination of lipids, proteins, carbohydrates, and minerals. ${ }^{23}$ The liver is divided into roughly hexagonal units, designated as hepatic lobules. They are composed of liver parenchymal cells (hepatocytes), arranged into plates, lined by a vascular network of sinusoids that empty into a central vein, and a portal triad at the lobule's corners. Figure 1 presents a typical human liver histology section:

All the liver tissue samples used in this study were collected from three adult patients undergoing partial liver resection at the Portuguese Oncology Institute of Porto, Portugal. After collection of the specimens from patients, only healthy fragments were made available for our studies, since the rest was used for clinical diagnosis and analysis at the Portuguese Oncology Institute of Porto. These patients, two men and a woman with ages between 41 and 61, have signed a written consent previous to surgical procedures allowing for subsequent use of surgical specimens for diagnostic and research purposes.

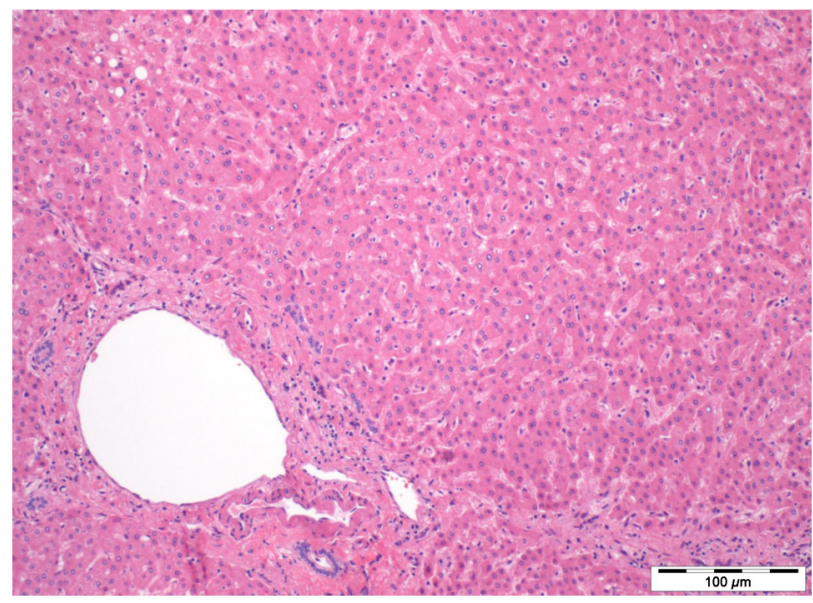

Fig. 1 Human liver histology section, showing hepatocyte plates and a portal triad (lower left) (HE, 100x).

Such agreement has been approved by the Ethics Committe of the Portuguese Oncology Institute of Porto, Portugal.

The samples to be used in the RI measurements had approximated rectangular shape $\left(\sim 4 \times 3 \mathrm{~cm}^{2}\right)$ and $\sim 1 \mathrm{~cm}$ thickness. These samples were flattened on one side to adhere perfectly to a prism surface during measurements with the total internal reflection method. The samples to be used in the spectral $T_{\mathrm{c}}$ measurements were prepared with a $0.5-\mathrm{mm}$ thickness, and an approximate circular form $(\phi \cong 1 \mathrm{~cm})$. To prepare the liver samples with these conditions, a cryostat from Leica ${ }^{\mathrm{TM}}$, model CM 1850 UV was used.

\subsection{RI Measurements}

To measure the RI of liver at discrete wavelengths, we have used the total internal reflection method, ${ }^{24-28}$ with a setup as represented in Fig. 2.

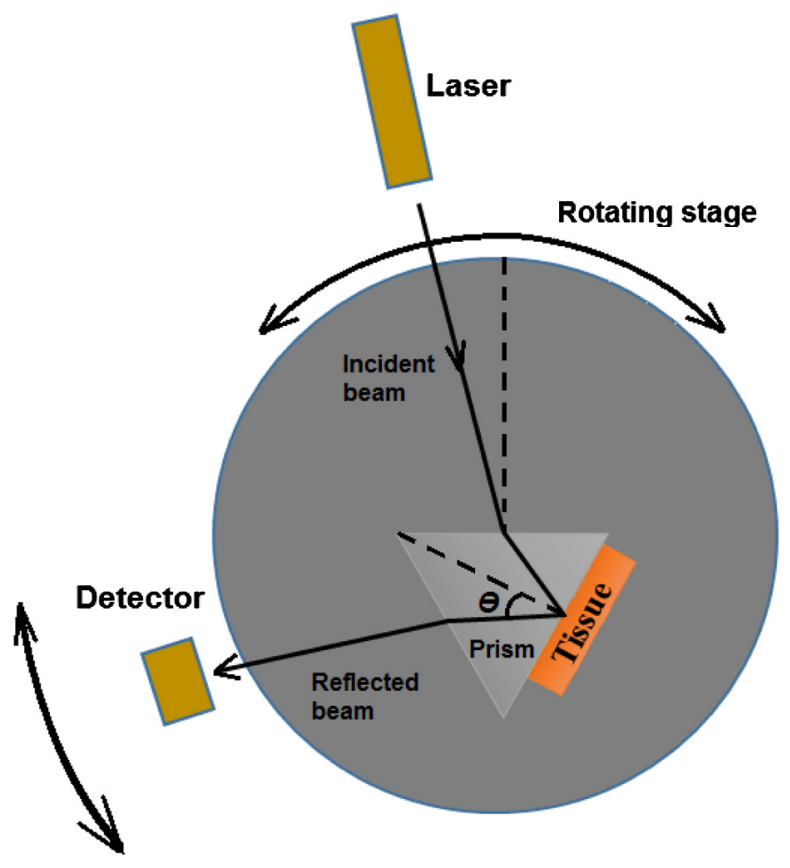

Fig. 2 Setup used on internal reflection measurements. 
In Fig. 2, the detector is connected to an electrical multimeter to measure the electrical potential at different angular positions. Using this method, angular reflectance measurements at the prism/tissue interface were performed with lasers at wavelengths of $401.4,532.5,668.1,782.1,820.8$, and $850.7 \mathrm{~nm}$. Measurements were made with all lasers from three liver samples with $1 \mathrm{deg}$ resolution. All the lasers are diode lasers from Edmund Optics ${ }^{\mathrm{TM}}$, with the exception of the 668.1-nm laser, which was acquired from Melles Griot ${ }^{\mathrm{TM}}$. The dispersion prism represented in Fig. 2 is an equilateral prism made of SCHOTT N-SF11 glass, and it was also supplied by Edmund Optics $^{\mathrm{TM}}$. This type of glass has an RI with a wavelength dependence described by the Sellmeyer equation ${ }^{29}$

$n^{2}-1=\frac{K_{1} \lambda^{2}}{\lambda^{2}-L_{1}}+\frac{K_{2} \lambda^{2}}{\lambda^{2}-L_{2}}+\frac{K_{3} \lambda^{2}}{\lambda^{2}-L_{3}}$.

Reference 29 indicates the Sellmeyer coefficients for this type of glass as: $K_{1}=1.7376, K_{2}=0.3137, K_{3}=1.8988, L_{1}=$ $0.0132, L_{2}=0.0623$, and $L_{3}=155.2363$. The RI described by Eq. (1) shows decreasing behavior with wavelength.

Since the angle of the reflected beam could only be measured outside the prism at the prism/air interface, we have used the Snell-Descartes equation [Eq. (2)] to convert between this angle $(\alpha)$ and the reflected angle at the prism/tissue interface $(\theta)^{30}$

$\theta=\beta-\arcsin \left[\frac{1}{n_{1}} \times \sin (\alpha)\right]$,

where $\beta$ is the internal angle of the prism (60 deg for our prism) and $n_{1}$ is the RI of the prism at the wavelength of the laser in use. For each laser we used, this value was retrieved from the graph in Fig. 3.

As a result of each set of measurements with a particular laser, we obtained a reflectance curve for the tissue/prism interface. Such a reflectance curve was calculated according to

$R(\theta)=\frac{V(\theta)-V_{\text {noise }}}{V_{\text {laser }}-V_{\text {noise }}}$,

where $V(\theta)$ represents the potential measured at angle $\theta, V_{\text {noise }}$ is the potential measured with background light, and $V_{\text {laser }}$ represents the potential measured directly from the laser.

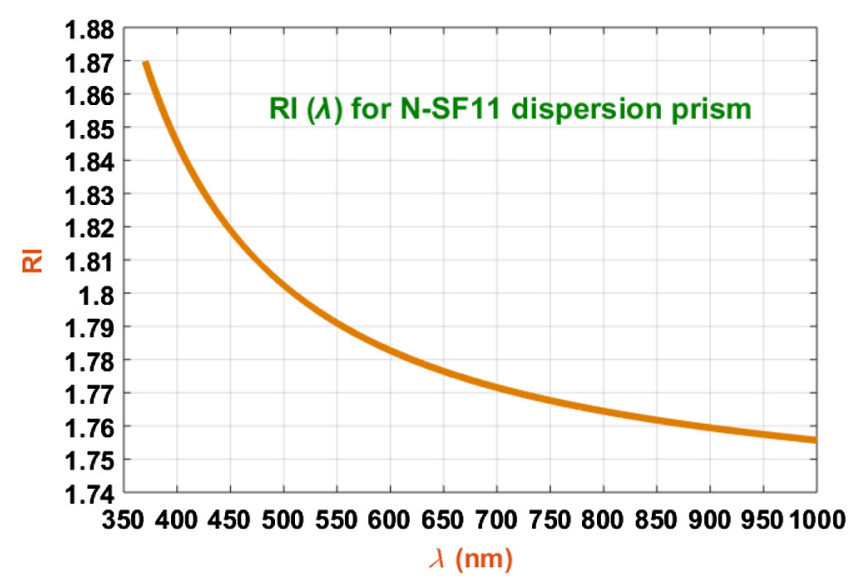

Fig. 3 Wavelength dependence for the RI of the prism used in the RI measurements.
A representation of the reflectance at the prism/tissue interface $[R(\theta)]$ shows an increase in reflectance from a lower to a top value as a function of the incidence angle.

By calculating the first derivative of the reflectance curve, we were able to identify a strong peak that corresponds to the critical angle $\left(\theta_{c}\right)$ at the prism/tissue interface. The first derivative of the reflectance curve is calculated according to

$\operatorname{deriv}(\theta)=\frac{\operatorname{Ref}\left(\theta_{i}\right)-\operatorname{Ref}\left(\theta_{i-1}\right)}{\theta_{i}-\theta_{i-1}}$,

where $\operatorname{Ref}\left(\theta_{i}\right)$ represents the reflectance at a particular angle, $\theta_{i}$, and $\operatorname{Ref}\left(\theta_{i-1}\right)$ represents the reflectance at the previous angle $\theta_{i-1}$.

After determining $\theta_{\mathrm{c}}$ from the curve calculated with Eq. (4), the RI of the tissue $\left(n_{\mathrm{t}}\right)$ is calculated with

$n_{\mathrm{t}}(\lambda)=n_{1}(\lambda) \times \sin \left(\theta_{\mathrm{c}}\right)$.

In Eq. (5), $n_{1}(\lambda)$ represents the RI of the prism at the particular wavelength of the laser used to obtain the measurements. Since three sets of measurements were made for each laser, the resulting three RI values of the tissue at each wavelength were averaged.

Since the lasers we used do not cover the entire wavelength range from 400 to $1000 \mathrm{~nm}$, some literature data were used to extrapolate from 850 to $1000 \mathrm{~nm} \cdot{ }^{31}$ All the calculations and the procedure used in this extrapolation are presented in Sec. 3.

\section{3 $T_{\mathrm{c}}$ Measurements During Treatments with Glycerol Solutions}

According to our previous studies with different tissues and OCAs, ${ }^{11,16}$ the free water content in a biological tissue can be determined, based only on temporal $\left[T_{\mathrm{c}}(t)\right]$ measurements made during treatments with solutions containing different OCA concentrations.

To obtain the free water content in human liver, we have performed similar measurements using the setup represented in Fig. 4.

The chamber that is represented in Fig. 4 was constructed in black hard-plastic and contains a transparent glass at the bottom to allow the passage of the collimated light beam. The light beam, with $1 \mathrm{~mm}$ in diameter, crosses the tissue sample at the center of the chamber and exits at the top to be delivered to the spectrometer. The four black rectangles that are placed above and below the tissue represent two rings that are used to fix the sample at the center of the chamber. This setup was used to measure the $T_{\mathrm{c}}$ spectra for natural tissue and during treatment, after filling the chamber with the treating solution. Optical fiber cables are used between the lamp and the chamber and between the chamber and the spectrometer and some appertures are placed on both sides to guarantee beam diameter equal to $1 \mathrm{~mm}$ all the way.

Using this setup, we have measured the $\left[T_{\mathrm{c}}(t)\right]$ spectrum from 10 liver samples in natural state and from several samples during treatment with aqueous solutions having the following glycerol concentrations: $20 \%, 25 \%, 30 \%, 35 \%, 40 \%, 45 \%$, $50 \%, 55 \%$, and $60 \%$. For the treatments with each glycerol concentration, three studies were made, meaning that 27 liver samples were used in the studies during treatments with glycerol solutions. All samples to use in these measurements were prepared with $0.5-\mathrm{mm}$ thickness. The average natural $T_{\mathrm{c}}$ 
To spectrometer

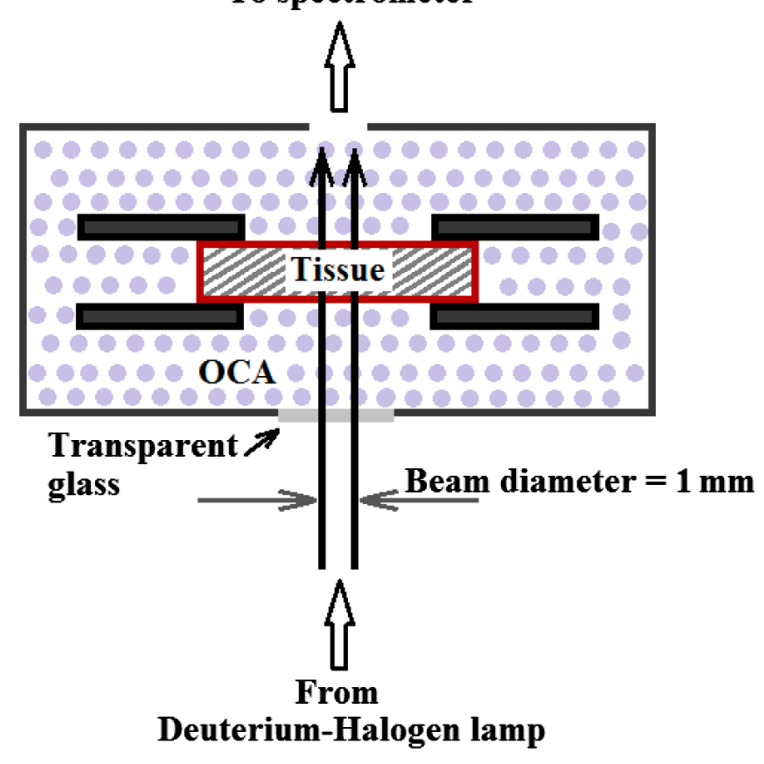

Fig. $4\left[T_{\mathrm{c}}(t)\right]$ measuring setup.

spectrum and average time dependencies obtained during treatments are presented in Sec. 3 .

\subsection{Calculation Procedure}

Considering the $\left[T_{\mathrm{c}}(t)\right]$ spectra measured during each particular treatment, we have selected the spectral range between 600 and $800 \mathrm{~nm}$ and calculated the mean time dependence for individual wavelengths within this range. This range is selected due to the almost linear $T_{\mathrm{c}}$ increase that the natural liver presents (see mean natural liver $T_{\mathrm{c}}$ spectrum at the beginning of Sec. 3). According to similar spectra observed for other tissues, muscle ${ }^{32}$ and colorectal mucosa tissues, ${ }^{11}$ tissue scattering in this range should be significantly greater than absorption.

The OCA diffusion into the interstitial space of the tissue is approximated by a free diffusion model. Since $T_{\mathrm{c}}$ measurements made during treatment are sensitive to the OCA diffusion, these measurements can be fitted with an equation that describes the free diffusion approximation, at least within the time range where this approximation is valid ${ }^{5,11,32}$

$T_{\mathrm{c}}(\lambda, t) \cong\left[1-\exp \left(-\frac{t}{\tau}\right)\right]$.

A graph was created to represent the time dependencies for the selected wavelengths for each treatment. From this graph, we identified the time range where Eq. (6) is valid and data for longer times is neglected. For each particular wavelength, the trimmed $T_{\mathrm{c}}$ time dependence is displaced verticaly to have $T_{\mathrm{c}}=0$ at $t=0$ and then normalized to the highest value. The final step is to fit the arranged time dependence with a curve described by Eq. (6).

When the fitting of experimental data is made, the diffusion time, $\tau$ for a particular wavelength and treatment is identified. The various values of $\tau$ obtained for the individual wavelengths are averaged to determine the mean diffusion time for that particular treatment.

Since this entire procedure is to be made for each treatment with a particular glycerol concentration, we have used a software application that we have developed for this purpose, ${ }^{33}$ which authomatizes the calculation procedure. This application uses MATLAB's CFTOOL to estimate the $\tau$ values while fitting the discrete time dependence data with a curve described by Eq. (6). After determining all the mean $\tau$ values for all treatments, the application generates a graph with the $\tau$ values represented as a function of the glycerol concentration in the treating solution, along with a smooth spline to fit the discrete data. From this graph, we can identify the maximal $\tau$ value that is observed at an optimal solution that contains the same water as the free water content in the liver. Section 3 contains graphs relative to the essential steps of this calculation.

Considering the RI values of liver tissue obtained for discrete wavelengths between 400 and $850 \mathrm{~nm}$, we had to calculate the RI dispersion for this tissue. We also wanted to extrapolate the dispersion to $1000 \mathrm{~nm}$. To do this extrapolation, we considered the discrete RI values for human liver collected by another group. $^{31}$ To obtain the correct dispersion, we have used CFTOOL in MATLAB. We fitted the discrete RI data with curves described by the usual equations for biological tissues, as described in the literature: ${ }^{34,35}$ Cauchy [Eq. (7)], Cornu [Eq. (8)], and Conrady [Eq. (9)] equations to verify which one provides the best fit

$n_{\text {liver }}(\lambda)=A+\frac{B}{\lambda^{2}}+\frac{C}{\lambda^{4}}$,

$n_{\text {liver }}(\lambda)=A+\frac{B}{(\lambda-C)}$,

$n_{\text {liver }}(\lambda)=A+\frac{B}{\lambda}+\frac{C}{\lambda^{3.5}}$.

While testing these equations to fit our data, we observed that both Cornu and Conrady equations provide the best fitting with corresponding $R$-square values of 0.999 and 0.998 . Consequently, we selected Cornu equation [Eq. (8)] to describe liver dispersion curve.

After determining the RI dispersion for human liver, we calculated the dispersion curves for the dry matter and scatterers. Since we have performed our entire measurements having a temperature range between $19^{\circ} \mathrm{C}$ and $21^{\circ} \mathrm{C}$, we collected the water dispersion between 400 and $1000 \mathrm{~nm}$ for $20^{\circ} \mathrm{C}$ from the literature. ${ }^{36}$ Considering the total water content of $74.5 \%$ in human adult liver, ${ }^{23}$ we subtracted the water contribution from liver dispersion to obtain the dispersion for dry matter. Considering the estimated free water content in liver, we subtracted its contribution from the liver dispersion to obtain the dispersion curve for scatterers. In these calculations, we used corrected versions of the Gladstone and Dale equation ${ }^{20}$

$$
\begin{aligned}
& n_{\text {dry-matter }}(\lambda)=\frac{n_{\text {liver }}(\lambda)-n_{\text {water }}(\lambda) f_{\text {water-total }}}{1-f_{\text {water-total }}}, \\
& n_{\text {scatterer }}(\lambda)=\frac{n_{\text {liver }}(\lambda)-n_{\text {water }}(\lambda) f_{\text {water-free }}}{1-f_{\text {water-free }}} .
\end{aligned}
$$

In Eqs. (10) and (11), $f_{\text {water-total }}$ and $f_{\text {water-free }}$ represent the total and free water contents in liver tissue, respectively.

All the results from the sequential calculation steps are presented in Sec. 3. 


\section{Results and Discussion}

As indicated in the previous section, our research involves different measurement and calculation procedures. Consequently, the presentation of results is also structured, beginning with the RI measurements of liver in Sec. 3.1. Sections 3.2 and 3.3 present the results that lead to the estimation of the glycerol diffusion coeficient in liver, liver free water content, and the calculation of the dispersion curves of dry matter and scatterers in liver, respectively.

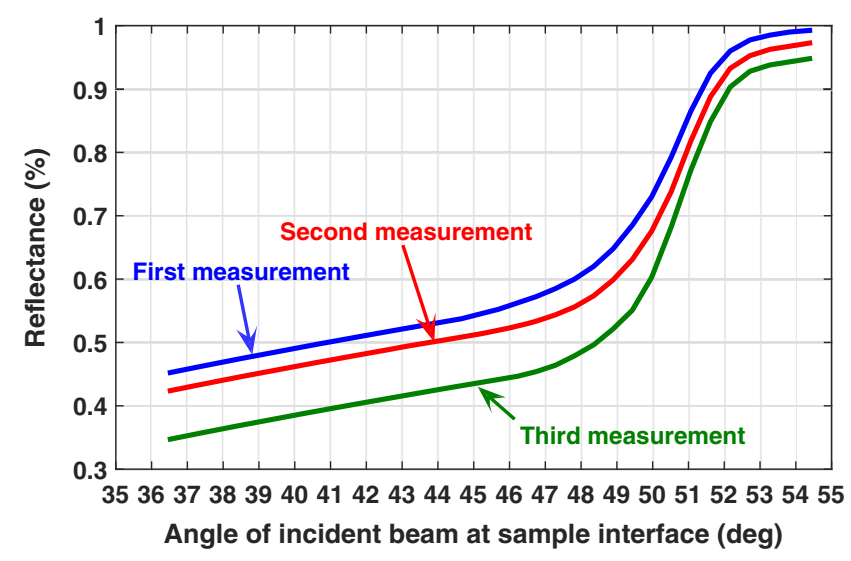

Fig. 5 Reflectance curves for 668.1-nm laser.

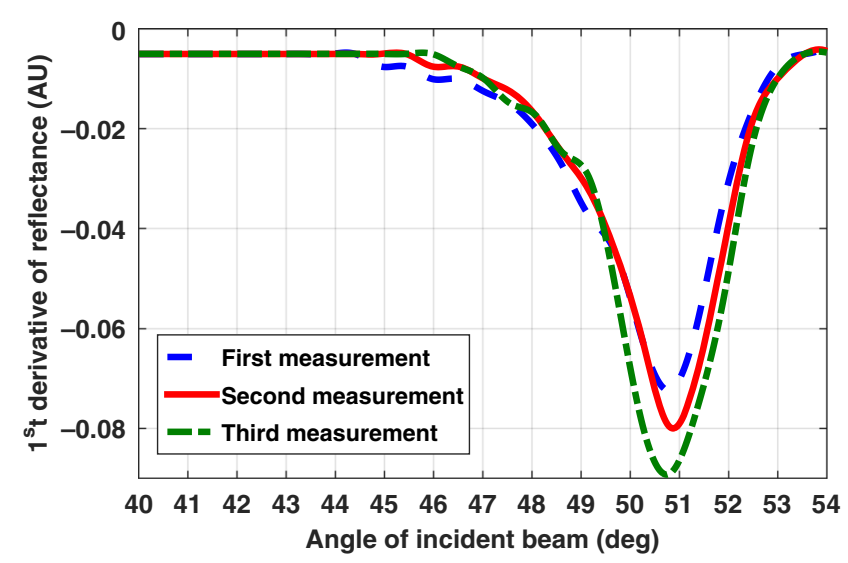

Fig. 6 First derivative curves of the reflectance measurements represented in Fig. 5.

\subsection{RI Dispersion for Human Liver}

To obtain the RI dispersion of human liver, we performed measurements with various lasers, using the total internal reflection method. As explained in Sec. 2.2, the liver samples to use in these measurements were prepared to have a flat surface, so they could be placed at the prism surface (see Fig. 2), without creating any air bubbles inbetween. For each of the lasers used, three angular reflectance curves at the prism/tissue interface were calculated from measurements with Eqs. (2) and (3). These curves are presented in Fig. 5 for the 668.1-nm laser.

Calculating the first derivative for each curve in Fig. 5, we obtained the curves presented in Fig. 6, where we can see a significant peak for each curve.

By identifying the angles for the peaks in the curves of Fig. 6, we could calculate the RI of the tissue for each set of measurements with the 668.1-nm laser. A mean value and correspondent standard deviation (SD) value were calculated for this wavelength. Such procedure was repeated for all the other lasers and the mean RI and SD values for all laser wavelengths are presented in Table 1.

The data in Table 1 and the reported RI values for wavelengths of 964 and $1551 \mathrm{~nm}^{31}$ were used to calculate the equation that better describes the RI dispersion for human liver. In this calculation, the RI values for 820.8 and 850.7 in Table 1 were neglected, since they are not well in line with the smooth RI decrease with wavelength. The experimental data in Table 1 and the calculated dispersion curve for human liver are presented in Fig. 7.

The dispersion curve represented in Fig. 7 is described by

$n_{\text {liver }}(\lambda)=1.3535+\frac{13.56}{(\lambda-37.24)}$.

The numerical values in Eq. (12) were obtained during the fitting of the experimental data with an $R$-square value of 0.999 . As can be seen in Fig. 7, the experimental RI values for 820.8 and $850.7 \mathrm{~nm}$ are a little higher than the calculated fitting curve. As in the case of colon mucosa tissues that we have previously studied, ${ }^{37,38}$ the increase in the RI at these wavelengths is evidence of lipid content in liver. Such evidence of lipids in human liver is not surprising since liver is reported to accumulate lipids. ${ }^{23,39,40}$ Since in this wavelength range (784 to $870 \mathrm{~nm}$ ) we have made measurements with only two lasers, it is not possible to make perfect characterization of the wavelength dependence created by lipids. Instead, we have represented the curve described by Eq. (12) in Fig. 7 for the entire wavelength

Table 1 Experimental RI data of healthy human liver at different wavelengths.

\begin{tabular}{|c|c|c|c|c|c|}
\hline$\lambda(\mathrm{nm})$ & First measurement & Second measurement & Third measurement & Mean RI & SD \\
\hline 401.4 & 1.3909 & 1.3914 & 1.3896 & 1.3906 & 0.0009 \\
\hline 532.5 & 1.3821 & 1.3780 & 1.3819 & 1.3807 & 0.0023 \\
\hline 668.1 & 1.3750 & 1.3756 & 1.3743 & 1.3750 & 0.0007 \\
\hline 782.1 & 1.3714 & 1.3737 & 1.3710 & 1.3720 & 0.0015 \\
\hline 820.8 & 1.3732 & 1.3708 & 1.3723 & 1.3721 & 0.0012 \\
\hline 850.7 & 1.3720 & 1.3726 & 1.3715 & 1.3720 & 0.0006 \\
\hline
\end{tabular}




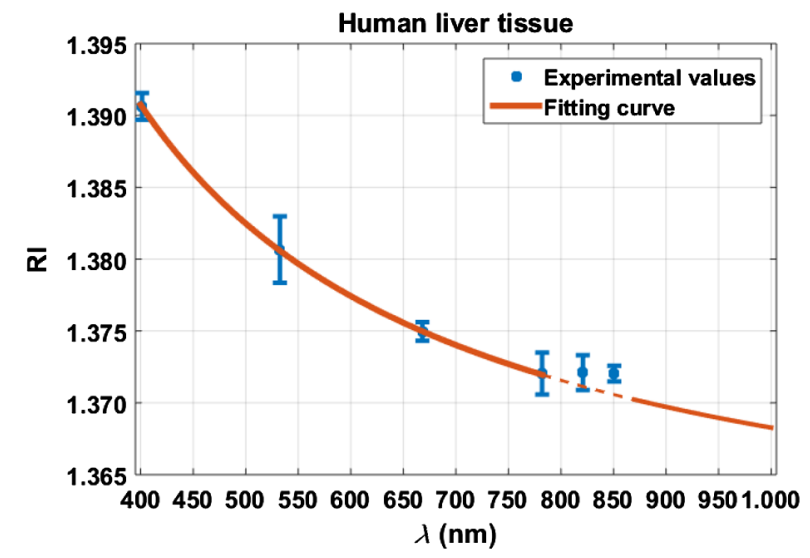

Fig. 7 Wavelength dependence of the RI of human liver between 400 and $1000 \mathrm{~nm}$.

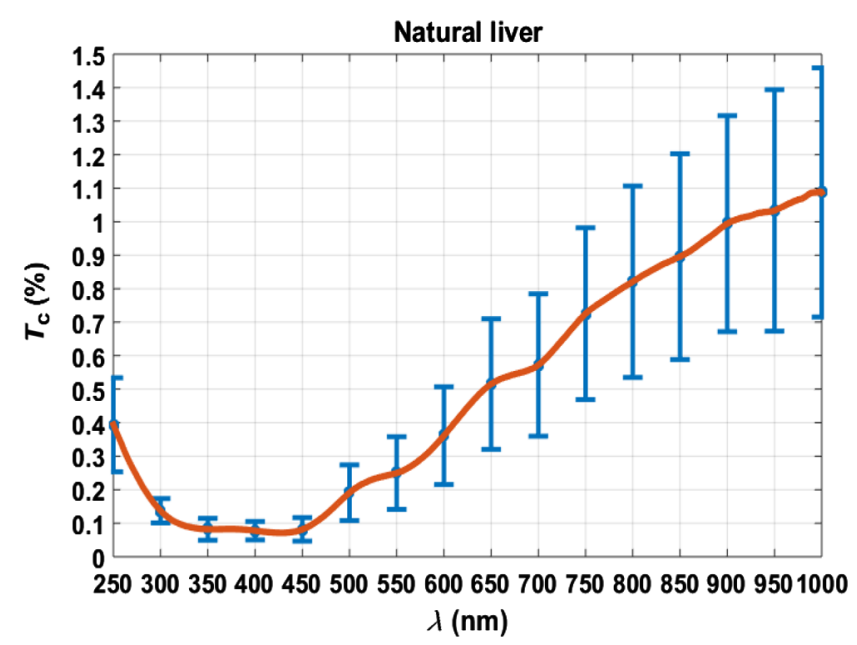

Fig. $8 T_{\mathrm{C}}$ spectrum of natural human liver between 250 and $1000 \mathrm{~nm}$.

range but with dashed lines between 784 and $870 \mathrm{~nm}$. The curve described by Eq. (12) for the entire range between 400 and $1000 \mathrm{~nm}$ will be used later to calculate the RI dispersion curves for dry matter and scatterers in liver tissue.

\subsection{Determination of the Free Water Content in Human Liver}

To proceed with the calculations of the dispersion curves of dry matter and scatterers in human liver, we need first to estimate the free water content in this tissue.

To obtain that value, we have performed spectral $T_{\mathrm{c}}$ measurements from liver samples under treatment with glycerol solutions. We will also use these measurements to calculate the diffusion coefficients of glycerol and water in liver that characterize the dehydration and RI matching mechanisms for these treatments. As indicated in Sec. 2.3, all samples used in these treatments had $0.5-\mathrm{mm}$ thickness and three treatments were made for each glycerol concentration in solution. The measurements performed in the three treatments with each glycerol concentration were averaged to create a mean spectral time dependence for each case. Figure 8 presents the average $T_{\mathrm{c}}$ spectrum of natural liver-average of 10 samples.

Figure 8 shows that only between 300 and $500 \mathrm{~nm}$ experimental spectra is not too dispersive. This range contains the
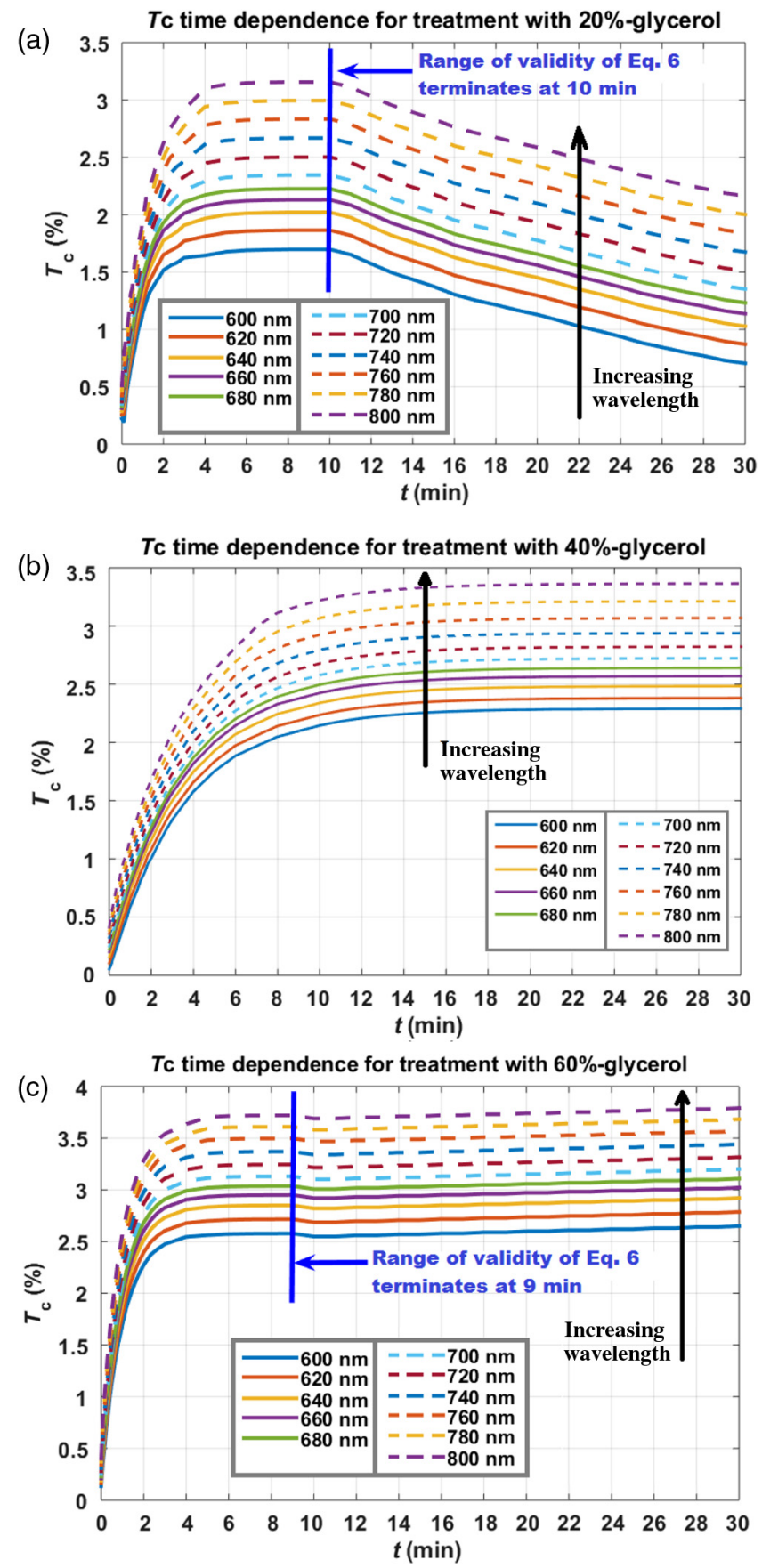

Fig. 9 Mean $T_{\mathrm{c}}$ time dependencies for wavelengths between 600 and $800 \mathrm{~nm}$ for treatments with: (a) $20 \%$-glycerol, (b) $40 \%$-glycerol, and (c) $60 \%$-glycerol.

hemoglobin absorption bands, meaning that tissue transparency is approximately the same for all samples used due to the presence of blood. All samples were prepared with 0.5 -mm thickness, but the data in Fig. 8 show that the samples considered were more or less transparent at shorter and longer wavelengths.

Considering now the OC treatments, Fig. 9 presents the mean time dependencies for the treatments with $20 \%-, 40 \%-$, and $60 \%$-glycerol solutions.

Similar behavior to the one presented in graphs of Fig. 9 was already observed in our previous studies with different tissues. ${ }^{11,32}$ As described in Sec. 2.4, we identified for each treatment the range of validity for Eq. (6). For the treatment with 
Table 2 Mean diffusion time of glycerol solutions in human liver.

\begin{tabular}{lcc} 
Glycerol concentration (\%) & Mean $\tau(\mathrm{s})$ & SD \\
\hline 20 & 69.0 & 3.9 \\
25 & 71.2 & 3.5 \\
30 & 80.9 & 5.4 \\
35 & 143.6 & 8.1 \\
40 & 211.2 & 2.8 \\
45 & 147.4 & 0.8 \\
50 & 91.2 & 1.2 \\
55 & 66.2 & 1.7 \\
60 & 57.2 & 0.8 \\
\hline
\end{tabular}

$20 \%$-glycerol, this range terminates at $10 \mathrm{~min}$. For the treatment with $40 \%$-glycerol, the range terminates at the end of the treatment, and for the treatment with $60 \%$-glycerol, it terminates at $9 \mathrm{~min}$. This means that the $T_{\mathrm{c}}$ data after $10 \mathrm{~min}$ in the treatment with 20\%-glycerol [Fig. 9(a)] and after 9 min in the treatment with $60 \%$-glycerol [Fig. 9(c)] were neglected for further data processing and calculations.

Similar graphs were generated by our software application ${ }^{33}$ for the treatments with other concentrations of glycerol. For each particular treatment, and after trimming the $T_{\mathrm{c}}$ time dependencies to the range of validity of Eq. (6), the $T_{\mathrm{c}}$ data were displaced to have $T_{\mathrm{c}}=0$ at $t=0$. The following step consisted of normalizing the trimmed time dependence data to its highest value. After these adjustements, we used CFTOOL in MATLAB ${ }^{\circledR}$ to fit the adjusted time dependence with a curve described by Eq. (6). With this fitting, we obtained the $\tau$ value for that particular time dependence that corresponds to an individual wavelength and an individual treatment. Averaging between all $\tau$ values for a single treatment, we obtained the mean and SD for $\tau$. This entire procedure was made for all treatments, and the calculated data are presented in Table 2.

Representing the data in Table 2 as a function of glycerol concentration in the treating solutions used in the study, we can adjust a spline to see the dependence. This graph is represented in Fig. 10.

For any particular treatment, the estimated $\tau$ value represents a combination of two fluxes: water flowing into (low glycerol concentrations) or out (high glycerol concentrations) and glycerol flowing only into the tissue. By calculating the fitting spline in Fig. 10, we see that it shows a maximum for a glycerol concentration of $40.0 \%$ in the treating solution. This slowest $\tau$ value indicates that due to the existence of an equilibrium between the water in the treating solution and the free water in the tissue, only glycerol flows in, without any water flowing in or out. This means that the free water content in human liver is $60.0 \%$. The slowest $\tau$ value in Fig. 10 (211.2 s) is the true diffusion time for glycerol in liver and it can be used to calculate the characteristic diffusion coefficient through $5,11,16,32$

$D_{a}=\frac{d^{2}}{\pi^{2} \tau}$.

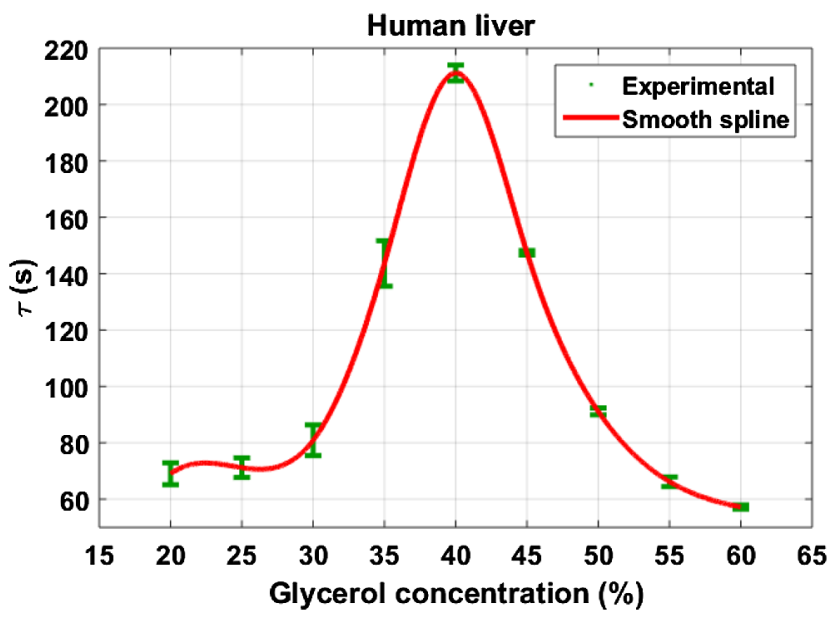

Fig. 10 Mean diffusion time as a function of glycerol concentration in solution.

Similarly, the fastest $\tau$ value in Fig. 10 (57.2 s) represents the diffusion time for water, and it can be used in Eq. (13) to calculate the diffusion coefficient of water during the dehydration mechanism. To perform these calculations, we need thickness measurements $(d)$ made during the treatments with $40 \%$ - and $60 \%$-glycerol. For an individual thickness set of measurements during a particular treatment, we have adopted the following procedure. The liver sample was placed in between two microscope glasses. Such a setup was introduced inside a precision micrometer to measure the global thickness of the glasses with the sample inside. The treating solution (40\%-glycerol or $60 \%$-glycerol) was injected inside the two glasses to initiate the treatment, and measurements were taken at each $15 \mathrm{~s}$ until 2 min of treatment and at each min after that. We obtained the sample thickness during treatment by subtracting the glasses thickness from the measurements performed. Three sets of measurements were made from three liver samples under treatment with $40 \%$-glycerol and other three from liver samples under treatment with $60 \%$-glycerol. Figure 11 presents the mean values for each treatment and the corresponding SD bars (in blue). The lines in Fig. 11 represent smooth splines that were created to interpolate each set of measured data.

Considering the data in Fig. 11, we retrieved the sample thickness of $0.41 \mathrm{~mm}$ from the spline that corresponds to the treatment with $40 \%$-glycerol at $211.2 \mathrm{~s}$ and calculated the diffusion coefficient for glycerol in human liver as $8.2 \times 10^{-7} \mathrm{~cm}^{2} / \mathrm{s}$. Similarly, the mean sample thickness when treated with $60 \%$-glycerol is $0.43 \mathrm{~mm}$, as retrieved from the spline in Fig. 11 for that treatment at 57.2 s. Using this value in Eq. (13), the diffusion coefficient for water in liver was calculated as $3.2 \times 10^{-6} \mathrm{~cm}^{2} / \mathrm{s}$. These diffusion coefficients are the values that characterize the $\mathrm{OC}$ mechanisms of tissue dehydration and RI matching for liver treatments with glycerol.

\subsection{Calculation of the Dispersion Curves for Scatterers and Dry Matter in Liver}

Considering the total water content in liver that has been reported $^{23}$ and the experimental data presented in previous sections, it is now possible to calculate the RI dispersion curves for the dry matter and scatterers.

To calculate the dispersion for the dry matter, we considered the liver dispersion curve in Fig. 7 and described by Eq. (12). 


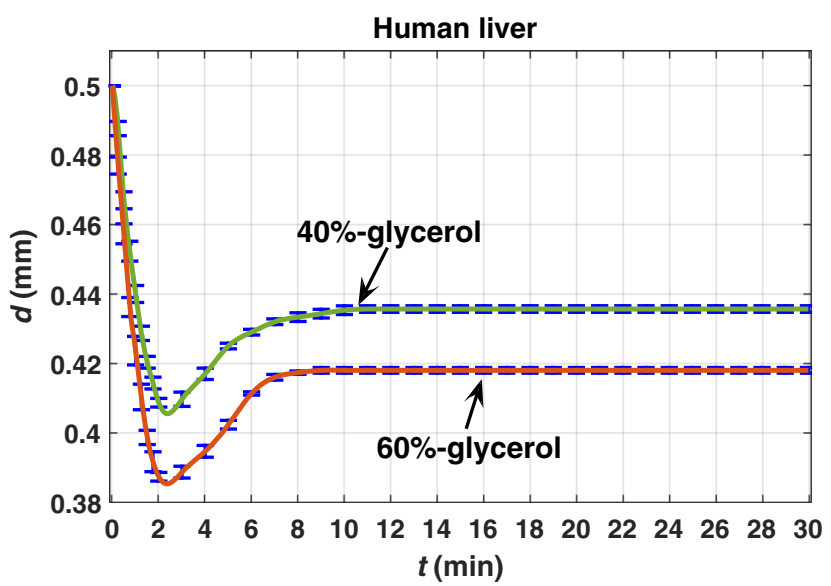

Fig. 11 Mean thickness time dependencies of liver samples under treatment with $40 \%$-glycerol and $60 \%$-glycerol.

We have also considered the total water content of $74.5 \%$ that is reported in the literature. ${ }^{23}$ Using these data in Eq. (10), we calculated the RI dispersion for dry matter. Using the same procedure, but now considering the estimated free water content of $60 \%$ in Eq. (11), we have calculated the dispersion curve for scatterers in human liver. Figure 12 contains both the calculated curves.

Figure 12 has two vertical axis to allow representing both curves toguether. As indicated by the ovals and arrows, the dispersion for scatterers is reported to the left vertical axis, whereas the dispersion of dry matter is reported to the right vertical axis. Although both curves show decreasing behavior with wavelength, the curve for dry matter presents higher values as it was expected due to the the fact that dry matter has no water. Since the dry matter in human liver contains lipids, proteins, carbohydrates, and minerals, the brown curve in Fig. 12 is a result of such particular combination. As an example, if similar studies are to be made in other liver samples that have excess of lipids (e.g., in steatotic liver), the calculated dispersion for dry matter might be compared to that depicted in Fig. 12 to identify that pathological condition. Liver accumulation of other materials that produce changes in RI can also be detected. The same dispersion may be useful for calculations in extreme

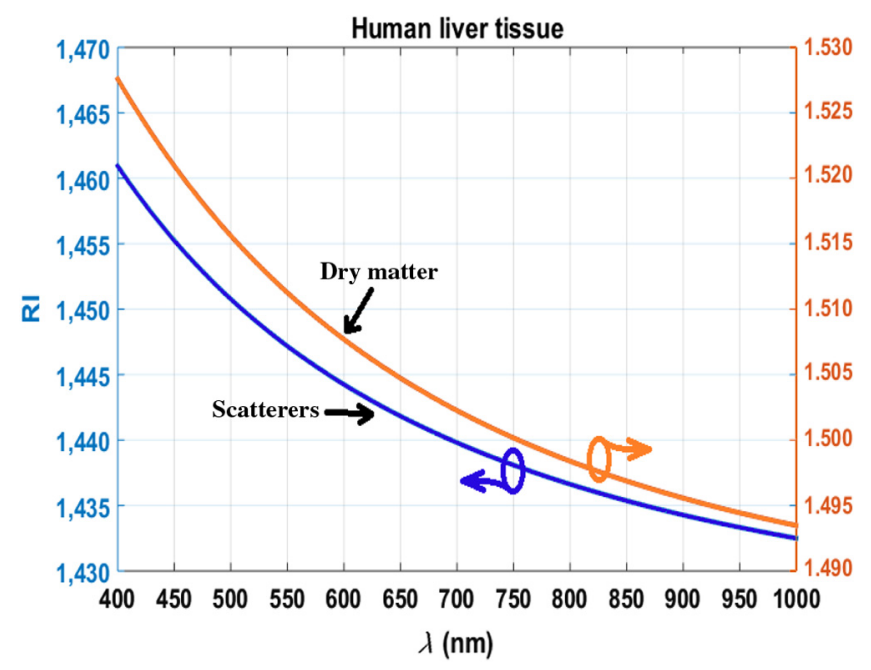

Fig. 12 Wavelength dependence of the RI of liver scatterers and dry matter.
OC treatments where bound water may convert into free water and new scatterers with smaller hydration are present.

The curve for scatterers in Fig. 12 is very important for OC treatment planning. If short-time treatments are to be planed, this curve will remain unchanged during treatment, due to the fact that only free water participates in the dehydration mechanism. Such curve can be used in calculations to quantify and characterize the RI matching mechanism that occurs due to the exchange of free water by OCA. Such calculations are described in the literature, ${ }^{5}$ and in one case that we have studied recently for skeletal muscle under treatment with glucose and ethylene glycol. ${ }^{19}$

\subsection{Possible Applications of Received Data}

During short-term immersion agent treatments, the RI matching mechanism occurs due to the exchange of free water in tissue fluids and the OCA in the treating solution. Bound water remains tightly connected with the other tissue components, a combination designated as tissue scatterers. Due to this water descrimination in biological tissues and its importance in OC treatments, the total water content of $74.5 \%$ for human liver previously reported in the literature is not sufficient to characterize OC treatments. In particular, the estimated $60.0 \%$ content for the free water and $14.5 \%$ for the bound water in human liver will allow one to perform prediction, quantification, and characterization of the RI matching mechanism at OC.

The knowledge of free/bound water content is also necessary for many other clinical applications, including tissue cryoprotection $^{41,42}$ and cancer diagnostics..$^{11,37,38,43}$ The OCAs are also mostly cryogenic agents and often used for cryoprotection of living tissues. Their strong osmotic properties and low-temperature freezing abilities are needed to prevent formation of ice crystals in order to keep tissue undamaged at low temperatures. ${ }^{41,42}$ Besides free and bound water contents, all these clinical technologies will be beneficial due to knowledge of the RI of scatterers and dry matter in tissues, including human liver, if optical monitoring is applied. The RI data for major tissue components may also be useful for different diagnostic purposes or to characterize long-term OC treatments when part of the bound water is converted into free water.

The calculated diffusion coefficients for glycerol and water in human liver are also important for cryoprotection applications or for OC treatment planning. Similar methods can be applied in studies with other OCAs, such as ethylene glycol, glucose, etc. to obtain similar data.

\section{Conclusions and Future Perspectives}

A simple multimeasurement method was used to estimate the diffusion coefficients of water and glycerol in human liver. These coefficients were calculated as $8.2 \times 10^{-7} \mathrm{~cm}^{2} / \mathrm{s}$ and $3.2 \times 10^{-6} \mathrm{~cm}^{2} / \mathrm{s}$, respectively, and they characterize the OC mechanisms for glycerol treatments. Similar studies can be performed with other OCAs to obtain similar data with application in cryoprotection of liver.

Using the same experimental data, the free water content in healthy human liver was also calculated as $60.0 \%$. This value and the reported total water content of $74.5 \%$ were used to calculate the dispersion curves for liver scatterers and dry mater. In these calculations, the measured liver dispersion and the reported water dispersion were also used. The calculated dispersions for scatterers and dry matter show a decreasing behavior 
with the wavelength. Due to bound water contribution in tissue scatterers, the correspondent dispersion shows smaller values than the ones seen for the dry matter in the entire wavelength range. The RI dispersion of scatterers decreases from 1.461 (at $400 \mathrm{~nm}$ ) to 1.432 (at $1000 \mathrm{~nm}$ ), whereas the dispersion of the dry mater decreases from 1.528 to 1.493 in the same wavelength range.

Considering that these studies have been made from tissue samples that were collected from patients with healthy livers, the dispersion curves received can be used as reference in future studies to detect excess of lipids or other liver components and identify those pathological conditions. The dispersion curve for dry matter may be useful for planing particular OC treatments or cryprotection of living tissues where strong osmotic OCAs can be used and where the bound to free water conversion is to be expected. The calculated dispersion curve for liver scatterers can now be used to plan and characterize OC treatments with different OCAs or to estimate the magnitude of the RI matching mechanism of OC for a particular treatment.

The methodology used in this research can be performed for other tissues to evaluate the free water content and calculate the RI dispersion curves for scatterers and dry matter. Since these data are very important for planning OC and cryogenic treatments, as well as for distinguishing normal and pathological tissues, we expect to conduct similar studies in other tissues soon.

\section{Disclosures}

No conflicts of interest, financial or otherwise, are declared by the authors.

\section{Acknowledgments}

This research was supported by the Portuguese Research Grant FCT/UID/EQU/04730/2013. V.V.T. was supported by the Russian Governmental Grant 074-U01 and RFBR Grant 17-02-00358.

\section{References}

1. T. Vo-Dinh, Ed., Biomedical Photonics Handbook, 2nd ed., Vol. 3, CRC Press, Boca Raton (2014).

2. I. J. Bigio and S. Fantini, Quantitative Biomedical Optics: Theory, Methods, and Applications, Textbook, Part of Cambridge Texts in Biomedical Engineering, Cambridge University Press, Cambridge (2016).

3. A. Sabouni, S. Noghanian, and S. Pistorius, "Effects of tissue composition on the accuracy of microwave breast tumour imaging," in World Congress on Medical Physics and Biomedical Engineering 2006, pp. 1489-1493, Springer, Berlin, Heidelberg (2007).

4. P. C. Miranda et al., "Tissue heterogeneity as a mechanism for localized neural stimulation by applied electric fields," Phys. Med. Biol. 52(18), 5603-5617 (2007).

5. V. V. Tuchin, Optical Clearing of Tissues and Blood, SPIE Press, Bellingham, Washington (2006).

6. L. Oliveira et al., "Optical characterization and composition of abdominal wall muscle from rat," Opt. Lasers Eng. 47(6), 667-672 (2009).

7. L. Oliveira et al., "Rat muscle opacity decrease due to the osmosis of a simple mixture," J. Biomed. Opt. 15(5), 055004 (2010).

8. V. Gilard et al., "Measurement of total water and bound water contents in human stratum corneum by in vitro proton nuclear magnetic resonance spectroscopy," Int. J. Cosmet. Sci. 20(2), 117-125 (1998).

9. C. Li, J. Jiang, and K. Xu, "The variations of water in human tissue under certain compression studied with diffuse reflectance spectroscopy," J. Innov. Opt. Health Sci. 6(1), 1350005 (2013).

10. D. Zhu et al., "Recent progress in tissue optical clearing," Laser Photonics Rev. 7(5), 732-757 (2013).
11. S. Carvalho et al., "Glucose diffusion in colorectal mucosa: a comparative study between normal and cancer tissues," J. Biomed. Opt. 22(9), 091506 (2017).

12. D. K. Tuchina et al., "Ex vivo optical measurements of glucose diffusion kinetics in native and diabetic mouse skin," J. Biophotonics 8(4), 332346 (2015).

13. E. A. Genina et al., "Optical clearing of biological tissues: prospects of application in medical diagnosis and phototherapy," J. Biomed. Photonics Eng. 1(1), 22-58 (2015).

14. E. A. Genina et al., "Tissue optical immersion clearing," Exp. Rev. Med. Dev. 7(6), 825-842 (2010).

15. L. Oliveira et al., "Optical clearing mechanisms characterization in muscle," J. Innov. Opt. Health Sci. 9(5), 1650035 (2016).

16. L. Oliveira et al., "The characteristic time of glucose diffusion measured for muscle tissue at optical clearing," Laser Phys. 23(7), 075606 (2013).

17. D. K. Tuchina et al., "Study of glycerol diffusion in skin and myocardium ex vivo under the conditions of developing alloxan-induced diabetes," J. Biomed. Photonics Eng. 3(2), 020302 (2017).

18. A. Sdobnov et al., "A comparative study of ex vivo skin optical clearing using two-photon microscopy," J. Biophotonics 10(9), 1115-1123 (2017).

19. L. Oliveira et al., "Skeletal muscle dispersion (400-1000 nm) and kinetics at optical clearing," J. Biophotonics e201700094 (2017).

20. D. W. Leonard and K. M. Meek, "Refractive indices of the collagen fibrils and extrafibrillar material of the corneal stroma," Biophys. $J$. 72(3), 1382-1387 (1997).

21. S. H. Chung et al., "In vivo water state measurements in breast cancer using broadband diffuse optical spectroscopy," Phys. Med. Biol. 53(23), 6713-6727 (2008).

22. H. Liu et al., "Dependence of tissue optical properties on solute-induced changes in refractive index and osmolarity," J. Biomed. Opt. 1(2), 200-211 (1996).

23. D. R. White et al., "The composition of body tissues (II) fetus to young adult," Br. J. Radiol. 64(758), 149-159 (1991).

24. J.-C. Lai et al., "Experimental measurement of the refractive index of biological tissues by total internal reflection," Appl. Opt. 44(10), 18451849 (2005).

25. H. Li and S. Xie, "Measurement method of the refractive index of biotissue by total internal reflection," Appl. Opt. 35(10), 1793-1795 (1996).

26. Q. Ye et al., "Measurement of the complex refractive index of tissuemimicking phantoms and biotissue by extended differential total reflection method," J. Biomed. Opt. 16(9), 097001 (2011).

27. H. Ding et al., "Determination of refractive indices of porcine skin tissues and intralipid at eight wavelengths between 325 and $1557 \mathrm{~nm}$," J. Opt. Soc. Am. A 22(6), 1151-1157 (2005).

28. Y. L. Jin et al., "Refractive index measurement for biomaterial samples by total internal reflection," Phys. Med. Biol. 51(20), N271-N379 (2006).

29. S. Glass, "Schott optical glass data sheets," http://refractiveindex.info (1 June 2016).

30. Q. W. Song et al., "Modified critical angle method for measuring the refractive index of bio-optical materials and its application to bacteriorhodopsin," J. Opt. Soc. Am. B 12(5), 797-803 (1995).

31. P. Giannios et al., "Visible to near-infrared refractive properties of freshly-excised human-liver tissues: marking hepatic malignancies," Sci. Rep. 6, 27910 (2016).

32. L. Oliveira et al., "Diffusion characteristics of ethylene glycol in skeletal muscle," J. Biomed. Opt. 20(5), 051019 (2015).

33. P. Peixoto et al., "Software development for estimation of optical clearing agent's diffusion coefficients in biological tissues," J. Biomed. Photonics Eng. 1(4), 255-269 (2016).

34. H. Ding et al., "Refractive indices of human skin tissues at eight wavelengths and estimated dispersion relations between 300 and $1600 \mathrm{~nm}$," Phys. Med. Biol. 51(6), 1479-1489 (2006).

35. Z. Deng et al., "Determination of continuous complex refractive dispersion of biotissue based on internal reflection," J. Biomed. Opt. 21(1), 015003 (2016).

36. G. M. Hale and M. R. Querry, "Optical constants of water in the $200 \mathrm{~nm}$ to $200 \mu \mathrm{m}$ wavelength region," Appl. Opt. 12(3), 555-563 (1973).

37. S. Carvalho et al., "Wavelength dependence of the refractive index of human colorectal tissues: comparison between healthy mucosa and cancer," J. Biomed. Photonics Eng. 2(4), 040307 (2016). 
38. S. Carvalho et al., "Comparative study of the optical properties of colon mucosa and colon precancerous polyps between 400 and $1000 \mathrm{~nm}$," Proc. SPIE 10063, 100631L (2017).

39. P. O. Kwiterovich, Jr. et al., "Glycolipids and other lipid constituents of normal human liver," J. Lipid Res. 11(4), 322-330 (1970).

40. H. Q. Woodard and D. R. White, "The composition of body tissues," Br. J. Radiol. 59(708), 1209-1218 (1986).

41. G.D. Elliott, S. Wang, and B. J. Fuller, "Cryoprotectants: a review of the actions and applications of cryoprotective solutes that modulate cell recovery from ultra-low temperatures," Cryobiology 76, 74-91 (2017).

42. J. M. Fox et al., "Methodology for reliable and reproducible cryopreservation of human cervical tissue," Cryobiology 77, 14-18 (2017).

43. S. H. Chung et al., "In vivo water state measurements in breast cancer using broadband diffuse optical spectroscopy," Phys. Med. Biol. 53, 6713-6727 (2008).

Isa Carneiro received her BSc degree in pathological anatomy in 2008 from Porto Polytechnic Institute and her MSc degree in oncology in 2012 from Porto University. She is a technician of pathological anatomy in the Department of Pathology of Portuguese Oncology Institute of Porto and is also a member of Cancer Biology and Epigenetics Group of Portuguese Oncology Institute of Porto Research Center. Her main research focus at the present is prostate cancer.

Sónia Carvalho is currently a fourth-year pathology resident at Portuguese Oncology Institute of Porto. She received her MD degree in 2012 from the Faculty of Medicine of Coimbra University, Portugal. In January 2013, she started supervised medical practice at Centro Hospitalar Lisboa Norte, Portugal. She participates in different medical and scientific activities within the scope of the residency. Her main research focus, at present, is colorectal carcinoma. She is a member of European Society of Pathology.

Rui Henrique received his $\mathrm{MD}$ and $\mathrm{PhD}$ degrees from the Abel Salazar Institute of Biomedical Sciences-University of Porto (ICBAS-UP), in 1992 and 2006. He is attending pathologist, subspecialized in hematopathology and uropathology in 2001, director of Department of Pathology in 2006, and senior researcher of Cancer Biology and Epigenetics Group in 2008 at Portuguese Oncology Institute of Porto, as well as invited full professor of ICBAS-UP. His research is focused on epigenetic alterations as cancer biomarkers in urological cancer.

Luís Oliveira is a professor and researcher at CIETI/Physics Department of the Polytechnic Institute of Porto-School of Engineering, Portugal. He received his $\mathrm{PhD}$ in biomedical engineering from Porto University, Portugal, in 2014. In recent years, he has authored and reviewed several papers in the field of tissue optical clearing. His main research interests are tissue optics, optical clearing and related applications, chemical diffusion in tissues and optical properties control to develop new clinical methods.

Valery V. Tuchin is a professor and head of optics and biophotonics at Saratov State University (National Research University of Russia) and several other universities. His research interests include tissue optics, laser medicine, tissue optical clearing, and nanobiophotonics. $\mathrm{He}$ is a fellow of SPIE and OSA, has been awarded honored science worker of the Russia, SPIE Educator Award, FiDiPro (Finland), Chime Bell Prize of Hubei Province (China), and Joseph W. Goodman Book Writing Award (OSA/SPIE). 